\title{
COLETA E AVALIAÇÃO DO SÊMEN DE CATETOS (Tayassu tajacu)
}

\author{
Deiler Sampaio Costa', Tarcízio Antônio Rego de Paula²
}

Biota Neotropica v5 (n2) - http://www.biotaneotropica.org.br/v5n2/pt/abstract?article+BN00705022005

Recebido em 06/04/2005

Revisado em 06/06/2005

Publicado em 01/07/2005

${ }^{1}$ Laboratório de Sanidade Animal da Universidade Estadual do Norte Fluminense

${ }^{2}$ Depto de Veterinária da Universidade Federal de Viçosa, tarcizio@ufv.br

Correspondência para: Prof. Dr. Deiler Sampaio Costa, Rua Jarbas Dias Pecly, 46/201, Falmboyant II, Campos dos Goyatacazes RJ, 28020-257 deiler@uenf.br

\begin{abstract}
The aim of this research was to test two electroejaculation protocols and to study the motility, vigor, concentration and morphologic alterations in captive collared peccaries semen. Six males with age between 10 and 18 years had used. The animals were anesthetized with zolazepan cloridrate and tiletamine cloridrate and the semen collected by electroejaculation. Average semen volume was 3,11 $+0,9 \mathrm{~mL}$, motility was $48.7 \pm 31.5 \%$, vigor, $2.1 \pm 1.4$ and concentration, $87.0 \pm 53.1 \times 10^{6} \mathrm{sptz} /$ $\mathrm{mL}$. It was concluded with this research that three sessions of 15 stimulations/12 volts was considered efficient for collared peccaries semen collection and that morphological spermatic results were compatible with testicular degeneration.
\end{abstract}

Key words: Tayassu tajacu, semen, electroejaculation.

\section{Resumo}

Objetivou-se com esta pesquisa testar dois protocolos de eletroejaculação e estudar a motilidade espermática progressiva, vigor, concentração e alterações morfológicas dos espermatozóides de catetos adultos criados em cativeiro. Utilizaram-se seis machos com idade entre 10 e 18 anos. Os animais foram anestesiados com cloridrato de zolazepan e cloridrato de tiletamina e em seguida coletou-se o sêmen com auxílio de um eletroejaculador. O volume médio do ejaculado foi 3,11 + 0,9mL, a motilidade espermática progressiva foi 48,7 $\pm 31,5 \%$, o vigor, 2,1 + 1,4 e a concentração espermática média, $87,0 \pm 53,1$ x $10^{6} \mathrm{sptz} / \mathrm{mL}$. Concluiu-se com esta pesquisa que a utilização de três sessões de 15 estímulos de 12 volts foi suficiente para coleta de sêmen de catetos anestesiados e que os resultados da morfologia espermática da maioria dos catetos trabalhados foram compatíveis com o quadro de degeneração testicular.

Palavras-chave: Tayassu tajacu, sêmen, eletroejaculação.

http://www.biotaneotropica.org.br 


\section{Introdução}

Os catetos (Tayassu tajacu) também conhecidos como caititus, porcos-do-mato ou pecaris, são animais gregários e rústicos que produzem carne e couro de excelente qualidade, para os quais existe grande demanda internacional. Membros da família Tayassuidae, os catetos se separaram da família Suidae a dezenas de milhões de anos (Bernirshchke, 1974). Portanto, apesar de serem bastante semelhantes ao porco doméstico e ao javali, os catetos não são porcos, diferindo dos mesmos em alguns aspectos, como por exemplo: pelo estômago que é dividido em quatro compartimentos (Cavalcante Filho, 1996), vesícula biliar ausente, membros pélvicos contendo três dígitos e pela presença de uma glândula de cheiro na região dorsal próximo à cauda, cuja secreção tem odor forte e coloração esbranquiçada (Sowls, 1974).

O cateto adulto tem em torno de 0,75 a $1,0 \mathrm{~m}$ de comprimento; 0,40 a 0,45m de altura e o peso variando de 14 a 30kg (Nowak \& Paradiso, 1983). Seus pelos são longos, ásperos e geralmente pretos com anéis brancos, conferindo uma pelagem acinzentada ao animal. Em seu dorso existe uma crina erétil composta de pelos que tendem a ser mais escuros e na região do pescoço destaca-se uma faixa de pelos brancos, dando um aspecto de colar (Sowls, 1984). Tais animais possuem pernas finas e a cabeça desproporcionalmente grande em relação ao restante do corpo. Praticamente não existe dimorfismo sexual nesta espécie, só sendo possível distinguir os sexos pela visualização do escroto dos machos, mesmo assim, quando observados à curta distância (Sowls, 1984).

A distribuição geográfica do cateto é bastante ampla, sendo encontrado naturalmente na América do Sul, na América Central e Sul dos Estados Unidos (Cabrera \&Yepes, 1940). Tal espécie vive em uma grande diversidade de habitat, desde regiões de florestas tropicais úmidas a regiões semi-áridas, conseguindo sobreviver mesmo em áreas devastadas (Sowls, 1984). Esta capacidade de sobrevivência desta espécie em diferentes condições se faz graças a adaptações fisiológicas e comportamentais, como por exemplo, a aceitação de uma longa lista de itens alimentares como frutas, folhas, raízes, cactáceos e tubérculos (Sowls, 1984). Em cativeiro esses animais também se adaptam facilmente a diferentes tipos de alimentação, sendo normalmente tratados com milho, mandioca, abóbora, banana, cana-de-açúcar triturada, silagem de milho, silagem de sorgo e ração comercial de suínos (Liva et al., 1989).

Em condições naturais os catetos vivem em grupos de cinco a 15 indivíduos, constituídos por animais jovens e adultos de ambos os sexos (Neal, 1959, Ellison \& Harwell, 1969, Schweinsburg, 1971). No Brasil, Miller (1930) relatou uma média de 12 indivíduos por grupo no estado do Mato Grosso. Normalmente, os grupos são estáveis e os seus membros não são expulsos, nascem e permanecem no mesmo grupo até a morte (Nogueira Filho, 1996, Nogueira Filho et al., 1999). Não há formação de bandos só de machos e nem haréns. No entanto, podem ocorrer fragmentações temporárias na época das chuvas, quando a vegetação fica mais densa, o que torna mais difícil a defesa contra predadores (Sowls, 1984). Esporadicamente, também podem ser registrados casos de animais isolados, geralmente indivíduos velhos ou doentes que não conseguem acompanhar o grupo (Schweinsburg, 1971).

A espermatogênese de catetos foi, recentemente, descrita por Costa et al. (2004), que relatou que tais animais possuíam o comprimento dos túbulos seminíferos por grama de parênquima testicular, a proporção volumétrica dos componentes dos testículos, a composição celular dos oito estádios do ciclo do epitélio seminífero, a capacidade de suporte de células de Sértoli e o rendimento geral da espermatogênese muito semelhante ao observado em suínos. Entretanto, pouco se sabe sobre as características seminais desta espécie.

Objetivou-se com esta pesquisa testar dois protocolos de eletroejaculação e estudar a motilidade espermática progressiva, vigor, concentração e alterações morfológicas dos espermatozóides de catetos adultos criados em cativeiro.

\section{Material e Métodos}

Utilizaram-se seis catetos com idade variando entre 10 e 18 anos e peso corporal em torno de $25 \mathrm{~kg}$. Os animais eram provenientes do Zoológico Municipal de Muriaé-MG (n = 3) e do Horto Zoobotânico São Francisco de Assis na cidade de Ubá-MG $(\mathrm{n}=3)$. Em ambos os casos os machos eram criados em cativeiro junto com fêmeas da espécie e alimentados com frutas, legumes e ração de suínos.

Após um prévio jejum alimentar e hídrico de 12 horas, os animais foram anestesiados utilizando-se zarabatana municiada com um dardo, confeccionado artesanalmente, contendo cloridrato de zolazepan associado ao cloridrato de tiletamina (Zoletil ${ }^{\circledR}$ Virbac) numa dosagem de $9,0 \mathrm{mg} / \mathrm{kg}$ (Calle \& Morris, 1999) e volume ajustado para 2,5mL. Passados no mínimo 20 minutos após a aplicação do anestésico, quando os animais já se encontravam em plano anestésico, procediam-se a tricotomia e higienização da região prepucial com água e sabão neutro e a exposição do pênis para que o sêmen fosse depositado diretamente no tubo coletor. Para a coleta de sêmen foi utilizado um eletroejaculador a bateria, equipado com uma probe retal de 2,0 cm de diâmetro e com dois eletrodos longitudinais.

Foram testados dois protocolos de eletroejaculação. O protocolo I constituiu de três sessões de 15 estímulos elétricos, intervaladas de três minutos de descanso. Na primeira sessão, foram cinco estímulos de três volts, cinco estímulos de quatro volts e cinco estímulos de cinco volts; na segunda, cinco de cinco volts, cinco de seis volts e cinco de sete volts; e na terceira sessão foram cinco estímulos de 
sete volts, cinco estímulos de oito volts e cinco estímulos de nove volts. Cada estímulo tinha duração de três a quatro segundos e era seguido do mesmo período de descanso (Evans, 1980, Basurdo-Kuba \& Evans, 1981).

O protocolo II constituiu de três sessões de 15 estímulos elétricos de 12 volts cada, aguardando-se um período de três minutos entre as sessões. A duração e o intervalo entre os estímulos elétricos foram os mesmo utilizados no protocolo I. As tentativas de coleta foram realizadas a cada 15-20 dias, alternando-se os protocolos utilizados. Assim sendo, cada animal foi submetido a oito tentativas de coleta, sendo quatro pelo protocolo I e quatro pelo protocolo II.

O sêmen foi coletado utilizando-se um funil acoplado a um tubo de ensaio graduado, com capacidade para $15 \mathrm{ml}$, que se encontrava dentro de uma garrafa contendo água a $37^{\circ} \mathrm{C}$. Foram analisados o volume, a motilidade espermática progressiva, o vigor, a concentração espermática e o pH do sêmen, segundo as recomendações do Colégio Brasileiro de Reprodução Animal (Henry \& Neves, 1998). As coletas foram realizadas durante os meses de fevereiro de março de 2003.

A análise estatística dos resultados foi puramente descritiva. As médias e desvios padrão dos dados obtidos neste experimento foram realizadas utilizandose o programa Excel.

\section{Resultados e Discussão}

O protocolo I que é indicado para coleta de sêmen em suínos, não foi eficiente para coletar sêmen dos catetos desta pesquisa em nenhuma das tentativas realizadas. Demonstrando que tal metodologia, apesar de ser eficiente em suínos, parece não ser indicada para catetos. Já o protocolo II foi eficiente para coleta de sêmen, fornecendo amostras suficientes para as análises utilizadas na rotina de um exame andrológico. Nos seis animais trabalhados os primeiros jatos ejaculados foram observados no final da primeira sessão de estímulos elétricos. Apenas na primeira das quatro coletas realizadas, no cateto três não houve ejaculação na primeira sessão de estímulos, tendo este animal apresentado os primeiros jatos de sêmen no final da segunda sessão de estímulos elétricos.

A associação de cloridrato de tiletamina e cloridrato de zolazepan, na dose utilizada $(9,0 \mathrm{mg} / \mathrm{kg})$, foi eficiente para produzir um plano anestésico que permitiu a realização de três sessões de estímulos elétricos sem que houvesse vocalização ou qualquer outro sinal clínico de sensibilidade dolorosa, sugerindo que tais procedimentos não causaram estresse excessivo nos animais.

O volume total de sêmen coletado variou muito entre os animais, média 2,98 + 2,29mL (Tab. 1), ao contrário do que foi relatado por Hellgren et al. (1989) que encontraram valores médios de 2,0 $\pm 0,2 \mathrm{~mL}$ por ejaculado. Da mesma forma, o volume de gel obtido, média $0,28 \pm 0,17 \mathrm{~mL}$, foi menor que o relatado por aqueles autores, $0,9 \pm 0,1 \mathrm{~mL}$.
A ocorrência de três frações no ejaculado de catetos, uma clara, uma rica em espermatozóides e uma fração de gel (Hellgren et al., 1989), também foi notada no presente experimento. Os primeiros jatos ejaculados eram normalmente constituídos das frações clara e rica, logo em seguida observava-se também a fração de gel. Essa característica foi notada em cinco dos seis animais experimentais. Apenas na primeira das quatro coletas realizadas, o ejaculado do animal 3 não apresentou a frações rica e de gel, neste caso a concentração espermática por mililitro foi muito inferior aos demais animais, sugerindo que a ejaculação foi incompleta.

Ejaculados de porcos domésticos coletados com auxílio de eletroejaculadores são caracterizados por um pequeno volume de gel (menos que 5,0 ml/ejaculado) e um baixo volume de líquido seminal (Clark, 1976, Vera Cruz, 1959) quando comparados com aqueles coletados pelo método da mão enluvada, comumente utilizado nesta espécie (Hancock \& Howell, 1959). Isso parece ter ocorrido nos catetos, uma vez que alguns autores relataram que se pode notar descarga vulvar de sêmen nas fêmeas após a cópula, podendo, por exemplo, atingir volumes de 45 e $80 \mathrm{ml}$, ressaltando ainda, a grande proporção de gel que é evidenciada nestes ejaculados (Sowls, 1966, Hellgren et al., 1989). Tal ocorrência associada a grande proximidade anatômica dos órgãos reprodutivos e com a espermatogênese de suínos (Costa et al., 2004, Sonner et al., 2004), sugerem que os catetos apresentam volume de ejaculado maior e contendo mais gel que o observado neste experimento.

Por sua vez, Basurdo-Kuba \& Evans (1981) em um estudo comparando os métodos de eletroejaculação e a técnica da mão enluvada para coleta de sêmen em varrões Hampshire, relataram que tanto a motilidade quanto o percentual de anormalidades das células espermáticas não diferiram entre os dois métodos. Tal observação sugere que o percentual de espermatozóides móveis, o vigor e a morfologia espermática do sêmen obtido pelo protocolo II são equiparáveis ao ejaculado normal dos catetos na mesma faixa etária. Portanto, a despeito de um menor volume ejaculado, os resultados das análises utilizadas na rotina do exame andrológico não seriam significativamente comprometidos quando se utilizasse essa técnica.

A concentração espermática média encontrada nos seis animais nas quatro coletas (média 87,0 + 53,1 x $10^{6}$ sptz/mL) foi bastante inferior a relatada por Hellgren et al. (1989) que obteve $371 \pm 30 \times 10^{6} \mathrm{sptz} / \mathrm{mL}$, trabalhando com catetos adultos, porém com idade muito inferior a dos animais desta pesquisa. Dentre as alterações no processo espermatogênico que ocorrem em animais velhos, podem-se destacar: o aumento do tecido conjuntivo intratesticular que pode levar a uma falha no processo de maturação destas células (Humphrey \& Ladds, 1975); redução da atividade metabólica das células envolvidas no processo (Leathem, 1977); redução do volume percentual de células da linhagem espermatogênica (Elcock \& Schoning, 1984); e redução 
Tabela 1 Características físicas do sêmen coletado por eletroejaculação e biometria testicular de catetos adultos

\begin{tabular}{|c|c|c|c|c|c|c|}
\hline \multirow{2}{*}{ Parâmetros } & \multicolumn{6}{|c|}{ Animais } \\
\hline & 01 & 02 & 03 & 04 & 05 & 06 \\
\hline Volume do ejaculado (ml) & $2,0 \pm 0,9$ & $4,9 \pm 1,8$ & $2,9 \pm 1,1$ & $3,1 \pm 0,2$ & $3,1 \pm 0,8$ & $2,7 \pm 0,6$ \\
\hline Volume de gel (ml) & $0,3 \pm 0,1$ & $0,3 \pm 0,1$ & $0,2 \pm 0,1$ & $0,3 \pm 0,1$ & $0,3 \pm 0,2$ & $0,3 \pm 0,1$ \\
\hline Motilidade (\%) & $87,5 \pm 2,9$ & $85,0 \pm 4,1$ & $17,5 \pm 6,5$ & $51,3 \pm 6,3$ & $32,5 \pm 9,6$ & $18,8 \pm 6,3$ \\
\hline Vigor $(1-5)$ & $4,5 \pm 0,6$ & $3,3 \pm 0,5$ & $1,0 \pm 0,0$ & $1,8 \pm 0,5$ & $1,0 \pm 0,0$ & $1,3 \pm 0,5$ \\
\hline Concentração (sptz/ ml) & $144,3 \pm 19,8$ & $119,8 \pm 13,6$ & $11,8 \pm 2,5$ & $132,5 \pm 10,7$ & $46,3 \pm 7,1$ & $67,5 \pm 9,3$ \\
\hline $\mathrm{pH}$ & $7,3 \pm 0,1$ & $7,6 \pm 0,1$ & $7,3 \pm 0,1$ & $7,2 \pm 0,2$ & $6,9 \pm 0,2$ & $7,1 \pm 0,2$ \\
\hline Biometria testicular $(\mathrm{cm})^{*}$ & & & & & & \\
\hline Testículo direito & $4,8 \times 3,1 \times 3,2$ & $5,1 \times 3,2 \times 3,4$ & $4,9 \times 3,1 \times 3,3$ & $5,1 \times 3,0 \times 3,3$ & $4,1 \times 3,5 \times 2,8$ & $5,2 \times 3,0 \times 3,8$ \\
\hline Testículo esquerdo & $5,0 \times 2,9 \times 3,3$ & $5,0 \times 2,9 \times 3,1$ & $5,1 \times 3,2 \times 3,4$ & $4,9 \times 2,9 \times 3,9$ & $4,5 \times 3,3 \times 2,9$ & $5,3 \times 2,9 \times 4,0$ \\
\hline
\end{tabular}

* comprimento x largura x espessura dos testículos

Tabela 2 Anormalidades morfológicas dos espermatozóides de catetos adultos

\begin{tabular}{|c|c|c|c|c|c|c|}
\hline \multirow{2}{*}{ Características Morfológicas } & \multicolumn{6}{|c|}{ Animais } \\
\hline & 01 & 02 & $03 *$ & 04 & 05 & 06 \\
\hline Acrossoma & & $3,5 \pm 1,4$ & $2,8 \pm 0,7$ & $3,0 \pm 2,0$ & & $1,5 \pm 0,1$ \\
\hline Piriforme & & $2,0 \pm 0,1$ & $4,3 \pm 1,1$ & $5,5 \pm 3,3$ & $8,0 \pm 1,3$ & \\
\hline Contorno anormal & $0,5 \pm 0,2$ & & $1,4 \pm 0,2$ & & & $2,0 \pm 0,3$ \\
\hline Cabeça isolada normal & & & $2,8 \pm 0,4$ & $0,5 \pm 0,2$ & $1,0 \pm 0,2$ & $1,5 \pm 0,2$ \\
\hline Abaxial, retroaxial, oblíquo & $0,5 \pm 0,1$ & & & & & \\
\hline Patol de peça intermediária & $2,0 \pm 0,3$ & $1,0 \pm 0,4$ & & & $1,5 \pm 0,4$ & $3,0 \pm 0,4$ \\
\hline Delgado & & & & $1,0 \pm 0,4$ & & $2,5 \pm 0,3$ \\
\hline Gigante, curto, largo, peq. normal & & & & $0,5 \pm 0,1$ & $0,5 \pm 0,2$ & \\
\hline Gota citoplasmática proximal & $8,0 \pm 1,2$ & $13,0 \pm 4,2$ & $24,3 \pm 6,8$ & $20,0 \pm 6,9$ & $26,0 \pm$ & $10,0 \pm 2,1$ \\
\hline Gota protoplasmática distal & $0,5 \pm 0,3$ & & $7,1 \pm 2,6$ & & $3,5 \pm 0,8$ & $2,0 \pm 0,7$ \\
\hline Cauda dobrada ou enrolada & $4,0 \pm 0,2$ & $6,0 \pm 2,8$ & $12,8 \pm 4,1$ & $10,0 \pm 3,7$ & $4,0 \pm 0,8$ & $14,0 \pm 4,2$ \\
\hline Total de anormalidades & $15,5 \pm 2,4$ & $25,5 \pm 5,9$ & $55,5 \pm 16,1$ & $40,5 \pm 9,5$ & $44,5 \pm 8,8$ & $36,5 \pm 7,6$ \\
\hline
\end{tabular}

* Percentual de anormalidades calculado a partir da contagem de 70 células

do diâmetro tubular e espessura do epitélio seminífero (Paula, 1992). Tais alterações se acumulam com o avançar da idade e estão ligadas ao próprio envelhecimento dos animais (Bishop, 1970).

Alterações degenerativas dos testículos, além de estarem relacionadas à idade avançada, também podem ser causadas por fatores como: deficiência nutricional, calor ou frio excessivo, radiação, lesões vasculares, traumatismos, dentre outras (McEntee, 1990). A degeneração testicular é a principal causa de infertilidade nos animais domésticos. Animais acometidos por esta patologia, normalmente, apresentam baixa concentração espermática, diferentes graus de queda da motilidade e alta incidência de espermatozóides com morfologia anormal (McEntee, 1990, Nascimento \& Santos, 1997). Sendo que tais alterações parecem ter sido responsáveis pelas características físicas e morfológicas do sêmen dos animais desta pesquisa (Tab. 1 e 2).
A morfologia espermática variou muito entre os machos, semelhante ao relatado por Hellgren et al. (1989), contudo, o percentual de células espermáticas anormais foi superior ao encontrado por aqueles autores. Tais diferenças eram previstas uma vez que os animais trabalhados na presente pesquisa tinham idade avançada. Dentre os defeitos menores, o tipo mais comum encontrado foi espermatozóides com a cauda dobrada ou enrolada. Esta patologia, geralmente, é devido à resposta das células espermáticas a condições ambientais extremas, ao choque térmico, trocas de $\mathrm{pH}$ e osmolaridade, agentes tóxicos, radiação ultravioleta, contaminação bacteriana e baixa freqüência copulatória (Crabo, 1986, Flowers, 1996). Sendo o estresse ambiental uma das prováveis causas desta alteração nos animais trabalhados. Uma vez que os mesmos estavam expostos a visitações constantes e os recintos em que se encontravam, não possuíam ambientação necessária para manutenção de catetos em cativeiro. 
Em relação aos defeitos maiores, a alteração mais observada foi a presença de gota citoplasmática proximal. A ocorrência desta alteração é um indicativo da imaturidade da célula espermática (Barth \& Oko, 1989) e um achado comum no ejaculado de animais com degeneração testicular (McEntee, 1990). A alta incidência de gota citoplasmática distal relatada por Hellgren et al. (1989) não foi encontrada nos animais deste experimento.

Ao examinar a consistência dos testículos dos catetos, percebeu-se que os animais 1, 2 e 6 apresentavam flacidez superficial e que nos animais 3, 4 e 5, aquele órgão possuía consistência aumentada, corroborando com os achados da morfologia seminal, que sugere um quadro de degeneração testicular. A influência de tais alterações sobre a fertilidade dos reprodutores, provavelmente deve ser semelhante à observada nos demais animais domésticos. Embora, tal parâmetro não tenha sido mensurado nesta pesquisa.

$\mathrm{O}$ pH do sêmen dos animais trabalhados (Tab. 1) foi semelhante ao encontrado para a grande maioria dos animais (Morrow, 1986). Por sua vez, a biometria testicular (Tab. 1) foi equiparável à relatada por Hellgren et al. (1989) trabalhando com 38 catetos adultos, com o relatado por Costa et al. (2004) e também por Sonner et al. (2004), sendo compatíveis com o encontrado em indivíduos sexualmente maduros.

Concluiu-se com esta pesquisa que a utilização de três sessões de 15 estímulos de 12 volts foi suficiente para coleta de sêmen de catetos anestesiados e que os resultados da morfologia espermática da maioria dos catetos trabalhados foram compatíveis com o quadro de degeneração testicular.

\section{Referências Bibliográficas}

BARTH, A.D. \& OKO, R.J. 1989. Abnormal morphology of bovine spermatozoa. Iowa State University Press, Ames.

BASURDO-KUBA, V.M. \& EVANS, L.E. 1981. Comparison of sperm-rich fractions of boar semen collected by electro ejaculation and the gloved-hand technique. J. Am. Vet. Med. Assoc. 178:985-986.

BENIRSHCHKE, K. 1974. Quest for the giant peccary: the chaco revisited. Zoonoz. 25:364-372.

BISHOP, M.W.H. 1970. Ageing and reproduction in the male. J. Reprod. Fertil. Suppl. 12:65-87.

CABRERA, A. \& YEPES, J. 1940. Historia natural ediar: mamiferos sud-americanos. Cia. Argentina de Editores. Buenos Aires.

CALLE, P.P. \& MORRIS, P.J. 1999. Anesthesia for nondomestic suids. In: Zoo \& Wild animal medicine current therapy 4 (folwler. M.E. \& Miller, R.E ed.). W.B. Saunders Company p. 639-646.

CAVALCANTE FILHO, M.F. Morfologia dos estômagos do queixada (Tayassu pecari) e do cateto (Tayassu tajacu) [Linnaues, 1789]. São Paulo, 1996. Dissertação Mestrado - Faculdade de Medicina Veterinária e Zootecnia, Universidade de São Paulo.
CLARK, T.L. 1976. Electroejaculation in the anesthetized and nonanesthetized boar. Proc. Int. Pig Vet. Soc. p.14.

COSTA, D.S., HENRY, M. \& PAULA, T.A.R. 2004. Espermatogênese de Catetos (Tayassu tajacu). Arq. Bras. Med. Vet. Zootec. 56:46-51.

CRABO, B.G. 1986. Factors affecting spermatogenesis and boar fertility In Current therapy in theriogenology (Morrow, D.A. ed.). W.B. Saunders. Philadelphia, p.975-978.

ELCOCK, L.H. \& SCHONING, P. 1984. Age-related changes in the cat testis and epididymis. Am. J. Vet. Res. 45:2380-2384.

ELLISON, J. E. \& HARWELL, F. 1969. Mobility and home range of collared peccary in southern texas. J. Wildl. Manage. 33:425-427.

EVANS, L.E 1980. Electroejaculation of the boar. In: MORROW, D.A. (Ed). Current therapy in theriogenology.. W.B. Saunders. Philadelphia, p.1037-1040.

FLOWERS, W.L. 1996. Semen evaluation, extension, packaging and transport methods. In: Annual Meeting of American Association of Swine Pratitioners. 27. Nashiville, Tennessee. USA. p.469-479.

HANCOCK, J.L. \& HOWELL, G.J.R. 1959. The collection of boar semen. Vet. Rec.71:664-665.

HELLGREN, E.C., LOCHUMILLER, M.S., AMOSS, JR. M.S. \& GRANT, W.E. 1989 Seasonal variation in serum testosterone, testicular measurement and semen characteristics in the collared peccary (Tayassu Tajacu). J. Reprod. Fertil. 85:677-686.

HUMPHREY, J.D. \& LADDS, P.W. 1975. A quantitative histological study of changes in the bovine testis and epididymis associated with age. Res. Vet. Sci. 19:135-141.

LEATHEM, J.H. 1977. Ageing and the testis. In The testis (Johnson, A.D., Gomes, W.R. \& Vandermark, N.L. ed) New York. Academic Press, v.4, p.547-563.

LIVA, H., MORAES, L.F.D., NOGUEIRA FILHO, S.L.G. \& LAVORENTI, A. 1989. Aspectos da alimentação do caititu (T. tajacu) em cativeiro. In Anais do Congresso Paulista de Iniciação Científica, I, Piracicaba.

LOW, W.A. 1970. The influence of aridity on reproduction of the collared peccary (Dicotyles tajacu) in Texas. Thesis Doctoral. University of British Columbia. Vancouver.

MCENTEE, K. 1990. Reproductive pathology in domestics mammals. San Diego: Academic Press.

MILLER, E.W. 1930. Notes on some mammals of southern Mato Grosso, Brazil. J. Mamm 11:18.

MORROW, D.A. 1986. Current therapy in theriogenology 2. W.B. Saunders Company.

NASCIMENTO, E.F. \& SANTOS, R.L. 1997. Patologia da reprodução dos animais domésticos. Guanabara-Koogan.

NEAL, B.J. 1959. A contribution on the life history of the collared peccary in Arizona. Amer. Midl. Nat. 61:177-190. 
NOGUEIRA FILHO, S.L.G. 1996. A organização social de queixadas (Tayassu pecari) e caititus (Tayassu tajacu) em cativeiro. Tese Doutorado. Instituto de Psicologia Universidade de São Paulo.

NOGUEIRAFILHO, S.L.G., NOGUEIRA, S.S.C. \& SATO, T. 1999. Estrutura social de pecaris (Mammalia, Tayassuidae) em cativeiro. Revista de Ecologia 1:89-98.

NOWAK, D. M.. \& PARADISO, J.L. 1983. Walker's Mammals of the World. 2 ed. The John Hopkins University Press, EUA. p.1184-1185.

PAULA, T.A.R. 1992. Estudo histológico quantitativo da atividade espermatogênica de cães s.r.d. em diferentes faixas etárias após a puberdade. Instituto de Ciências Biológicas. Dissertação Mestrado. Universidade Federal de Minas Gerais. Belo Horizonte.

SCHWEINSBURG, R.E. 1971. Home range movements and herd integrity of the collared peccary (Tayassu tajacu) J. Wildl. Man. 35:455-460.

SOONER, J.B., MIGLINO, M.A., SANTOS, T.C., CARVALHAL, R., ASSIS, A.C., MOURA, C.E.B. \& OLIVEIRA, M.F. Aspectos macroscópicos e moerfométricos dos testículos de catetos e queixadas. Biota Neotropica 4(2): $\underline{\text { http:// }}$ w w w.biotaneotropica.org, br/v 4 n $2 /$ abstract?article+BN03004022004

SOWLS, L.K. 1966. Reproduction in the collared peccary (Tayassu tajacu) In Comparative biology of reproduction in mammals (Rowlands, I.W., ed.) Zool. Soc. London. p.155-172.

SOWLS, L.K. 1984. The peccaries. The University of Arizona Press. Tucson, Arizona.

SOWLS, L.K. 1974. Social behavior of the collared peccary, Dicotyles tajacu, (L). In The behavior of ungulates and its relation to management (Geist, V., Walther, F. ed). Morges, Switzerland: IUCN. 24:144-165.

VERA CRUZ, N.C. 1959. Artificial insemination in pigs. I Semen collection by electrical stimulation in boars. Phillip. Agric. 43:225-235.

Título: Coleta e avaliação do sêmen de catetos (Tayassu tajacu).

Autores: Deiler Sampaio Costa, Tarcízio Antônio Rego de Paula

Biota Neotropica, Vol. 5 ( número 2): 2005

http://www.biotaneotropica.org.br/v5n2/pt/ abstract?article+BN00705022005

Recebido em 06/04/2005 - Revisado em 06/06/2005

Publicado em 01/07/2005

ISSN 1676-0603

http://www.biotaneotropica.org.br 